\title{
Efficacy and safety of percutaneous nephrolithotomy combined with negative pressure suction in the treatment of renal calculi: a systematic review and meta-analysis
}

\author{
Jiao Chen ${ }^{1 *}$, Xiaocong $\mathrm{Cai}^{2 \mathrm{2t}}$, Gang Wang ${ }^{3}$, Xiaofang $\mathrm{Chen}^{4}$, Danni $\mathrm{Lin}^{3}$ \\ ${ }^{1}$ Department of Operating Room, The Second Affiliated Hospital of Hainan Medical University, Haikou, China; ${ }^{2}$ Department of Urology, Danzhou \\ People's Hospital, Danzhou, China; ${ }^{3}$ Department of Urology, Central South University Xiang Ya School of Medicine Affiliated Haikou Hospital, \\ Haikou, China; ${ }^{4}$ Department of Anesthesiology, The Second Affiliated Hospital of Hainan Medical University, Haikou, China \\ Contributions: (I) Conception and design: J Chen; (II) Administrative support: X Cai; (III) Provision of study materials or patients: G Wang; (IV) \\ Collection and assembly of data: X Cai, X Chen; (V) Data analysis and interpretation: J Chen, D Lin; (VI) Manuscript writing: All authors; (VII) \\ Final approval of manuscript: All authors. \\ \#These authors contributed equally to this work. \\ Correspondence to: Danni Lin. Department of Urology, Central South University Xiang Ya School of Medicine Affiliated Haikou Hospital, Haikou \\ 570208, China. Email: lindanni138@163.com.
}

Background: With advances in medicine, there have been more and more ways to treat renal calculi in recent years. Percutaneous nephrolithotomy (PCNL) is a safe and effective treatment. The purpose of this paper is to study the efficacy and safety of PCNL combined with negative pressure suction in the treatment of renal calculi by meta-analysis.

Methods: The PubMed, EMBASE, Cochrane Library, Chinese Journal Full-Text Database, VIP, Wanfang Science and Technology Journal Full-Text Database, and Chinese Biomedical Literature Search databases were searched for articles related to the efficacy and safety of PCNL combined with negative pressure suction in the treatment of renal calculi from the establishment of the databases to October 2021. Endnote X9 software was first used to check and eliminate the articles, and the quality of the included articles were evaluated according to the risk of bias tool of Cochrane Collaboration. Stata 15.1 software was used to record the data. A meta-analysis was performed on the stone clearance rate, operation time, postoperative complications, postoperative fever, septic shock, intrapelvic pressure, and blood loss of PCNL combined with negative pressure suction in the treatment of renal calculi. The reliability of the results was assessed by a sensitivity analysis. Egger's linear test was used to test the publication bias of the articles.

Results: A total of 10 articles were included in the meta-analysis, and the total sample size of the study was 820. The meta-analysis showed that when PCNL combined with negative pressure suction was used to treat renal calculi, the stone clearance rate and the occurrence of septic shock of the test group did not differ significantly from that of the control group; the incidence rate of postoperative complications the operation time, the intraoperative bleeding and the postoperative fever of the test group was significantly better than that of the control group.

Discussion: Compared to the group without negative pressure, PCNL reduces the operation time, postoperative complications, postoperative fever, septic shock, and intraoperative blood loss without increasing the risk of septic shock.

Keywords: Percutaneous nephrolithotomy (PCNL); negative pressure suction; renal calculi; meta-analysis

Submitted Nov 17, 2021. Accepted for publication Jan 04, 2022.

doi: $10.21037 /$ tau-21-1067

View this article at: https://dx.doi.org/10.21037/tau-21-1067 


\section{Introduction}

Stones can occur in multiple systems of the human body, including the urinary system and digestive system (1). The most common site for stones is the urinary system (i.e., the kidneys). Clinical manifestations of stones include hematuria, lumbar and abdominal pain, nausea and vomiting, and in severe cases, sones can lead to urinary tract obstruction, which induces urinary tract infection and can even cause renal failure, leading to death (2). With the development of endoscopic technology and the progress of lithotripsy (the primary means of treating renal calculi) equipment, percutaneous nephrolithotomy (PCNL) has become the treatment of choice for complex renal calculi represented by cast stones (3); however, intraoperative renal pelvis pressure may cause sepsis, bacteremia and postoperative fever (4). PCNL is a method of crushing and removal of stones in the pyelonephrine or upper ureteral segment by percutaneous renal channels combined with pneumatic ballistic, holmium laser, or ultrasonic lithotripsy equipment. After years of development, percutaneous nephrolithotomy continuous improvement, puncture method from $\mathrm{X}$ line to ultrasound guidance and CT 3D reconstruction, percutaneous kidney channel from channel to small channel to standard channel and multiple channel combination, gravel from pneumatic lithotripsy to ultrasonic lithotripsy, di laser gravel and a new generation of ultrasonic clearance lithotripsy devices with negative pressure attraction device, from tube drainage to tubeless PCNL. In recent years, with the continuous development of science and technology and the progress of ideas, many scholars have suggested the use of negative pressure suction technology in PCNL to improve the stone clearance rate, reduce the operation time, reduce surgical complications, postoperative fever, septic shock, and intraoperative blood loss. These negative pressure techniques include ultrasonic lithotripsy instruments, Li's nephroscope (5), and the negative pressure stone evacuator. At present, there have been more and more relevant reports on PCNL combined with negative pressure suction in the treatment of renal calculi, but the results differ. Thus, this paper conducted a meta-analysis of relevant studies to explore the efficacy of PCNL combined with negative pressure suction in the treatment of renal calculi and to provide clinicians with diagnosis and treatment ideas and relevant theoretical support. We present the following article in accordance with the PRISMA reporting checklist (available at https:// tau.amegroups.com/article/view/10.21037/tau-21-1067/rc).

\section{Methods}

\section{Criteria for inclusion of literature in the study}

\section{Literature type}

All the included studies were randomized controlled trials (RCTs) on PCNL combined with negative pressure suction, and the languages were limited to Chinese and English.

\section{Participants}

Patients with renal calculi diagnosed by computed tomography (CT) or X-ray and confirmed by imaging studies were included in the study.

\section{Exclusion criteria}

Patients were excluded from the study if they had congenital urinary dysplasia, were aged $<18$ years, or had contraindications to nephroscopic lithotripsy. Studies were excluded if they were non-RCT studies (e.g., reviews, experience summaries, meta-analyses, cohort studies or case-control studies, individual or case studies), were basic studies with rats, rabbits, and other animals, or mainly examined other diseases.

\section{Description of intervention}

There were 2 groups of treatment modalities for kidney stones: (I) the experimental group, which used PCNL combined with negative pressure suction; and (II) the control group, which did not use negative pressure suction therapy.

\section{Outcome indicators}

The primary outcome indicators were the stone clearance. Different letter spacing, postoperative fever, and blood loss. The secondary outcome indicator was septic shock.

\section{Search strategy and literature identification}

The PubMed, EMBASE, Cochrane Library, Chinese Journal Full-Text Database (CNKI), VIP Journal FullText Database, Wanfang Science and Technology Journal Full-Text Database, and Chinese Biomedical Literature Search (CBM) databases were searched for articles on the efficacy of PCNL combined with negative pressure suction in the treatment of renal calculi from the establishment of the databases to October 2021. The English databases was 
searched using the keywords "Percutaneous Nephrolithotomy" and "Randomized controlled trial;" MeSH words in combination with free words were used.

\section{Literature screening and data extraction}

After the article retrieval was completed, Endnote X9 software was used for management. The automatic finding function of the software was used to find and exclude duplicate articles. Two reviewers independently screened the articles. The title abstract was read for the preliminary screening, and after any articles that did not meet the exclusion and inclusion criteria were excluded, the full text was then downloaded for further review. When the 2 reviewers' opinions differed, disagreements were resolved by discussion or by a $3 \mathrm{rd}$ reviewer.

The 2 reviewers independently completed the data extraction, and the following relevant data were extracted:

(I) Basic information on the article: title, author, name of publication journal, and publication time;

(II) Study characteristics: the total sample size of the study, and number of groups;

(III) Participant characteristics: age;

(IV) Intervention characteristics: the different intervention methods used for the control group and test group; and

(V) Assessment of results: stone clearance rate, operation time, postoperative complications, postoperative fever, septic shock, intrapelvic pressure, and blood loss.

\section{Bias of articles and evaluation analysis}

The assessment was performed using the Cochrane Collaboration's risk of bias tool (6). The assessment considered the generation of random sequences, allocation concealment, blindness to participants and implementers, blindness to outcome evaluators, selective reporting, and the completeness of the outcome data. The literature quality evaluation was completed independently by 2 reviewers, and if a disagreement arose, a 3 rd reviewer resolved the disagreement.

\section{Effect measurement}

The binary variables (i.e., stone clearance rate, postoperative complications, postoperative fever, and septic shock) were assessed using odds ratios (ORs) and their $95 \%$ confidence intervals (CIs). The continuous variables (i.e., operation time and blood loss) were assessed using standardized mean differences (SMD) and their 95\% CIs.

\section{Methods to address data loss}

If data were not provided in the article, the data were obtained by calculation if possible; if no data at all were provided, the author was contacted and the data were requested, and if the data were still not available, the article was excluded.

\section{Statistical Analysis and heterogeneity detection}

Stata15.1 was used for the analysis, and forest plots were used to represent the analysis results; $\mathrm{I}^{2}$ and $\mathrm{Q}$ tests were used to analyze literature heterogeneity, and $\mathrm{I}^{2}>50 \%$ or $\mathrm{P}<0.05$ was used to indicate a statistical difference in heterogeneity. If the test results showed statistical homogeneity $\left(\mathrm{I}^{2}<50 \%\right)$, the fixed-effects model was used to combine and analyze the data. If the test results showed statistical heterogeneity $\left(\mathrm{I}^{2}>50 \%\right)$, the heterogeneity was excluded by a subgroup analysis or sensitivity analysis according to the type of data. If heterogeneity still existed due to certain clinical homogeneity, the random-effects model was used to combine the data for analysis (7).

\section{Publication bias analysis}

The presence of publication bias was analyzed by Egger's linear test.

\section{Sensitivity analysis}

The sensitivity analyses were performed using the Influence Analysis tool provided by stata15.1.

\section{Results}

\section{Literature search results}

A total of 4,861 articles were initially found in this search. After duplicates were removed and the remaining articles were screened, a total of 10 articles were included in the meta-analysis. Figure 1 shows the literature screening process and results. 


\section{Identification of studies via databases and registers}

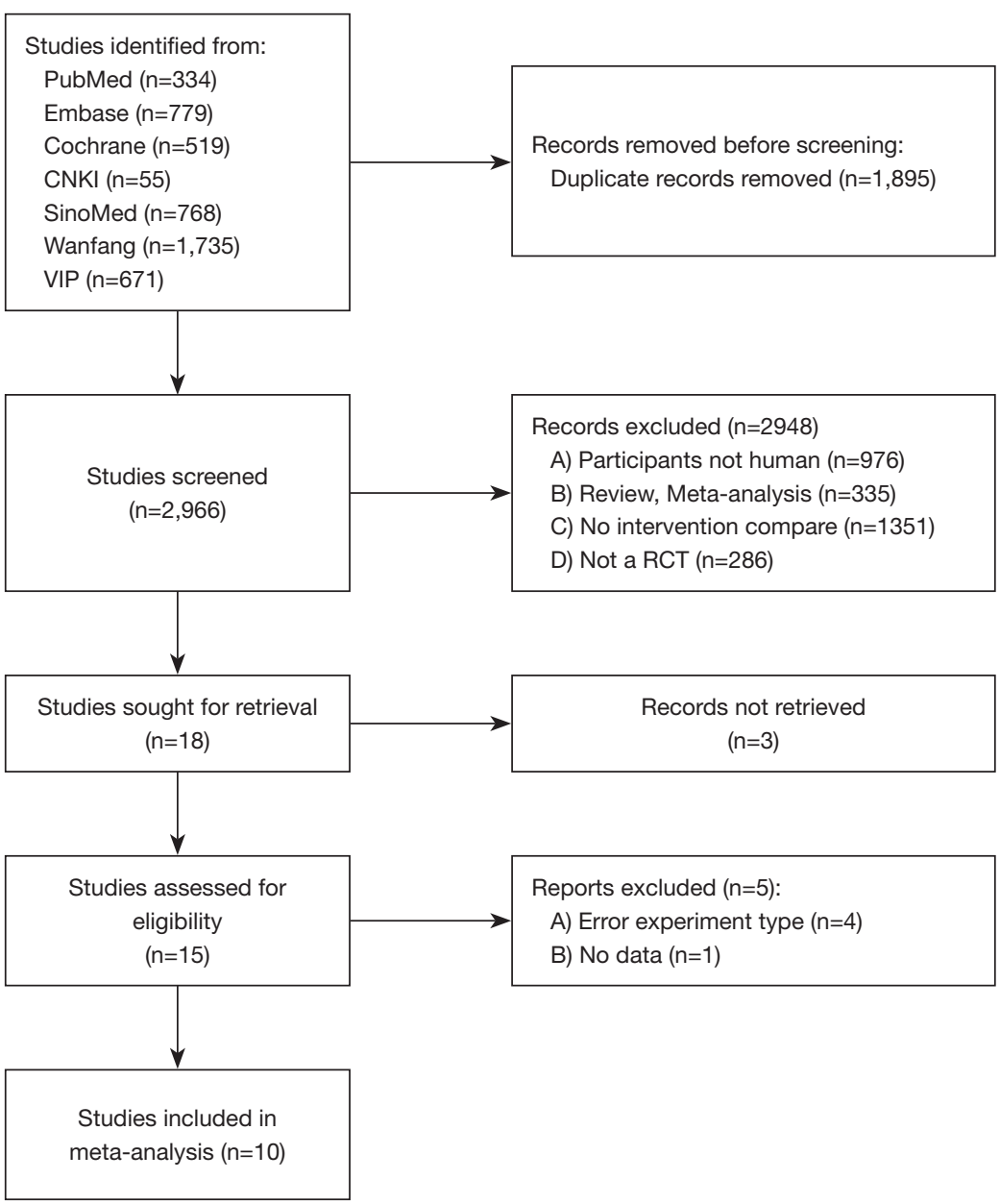

Figure 1 Literature screening flow chart.

\section{Basic characteristics of the included articles}

Ten articles (comprising a total of of 820 patients) were included in this meta-analysis. Basic information about the articles are shown in Table 1.

\section{Quality assessment}

According to the Cochrane Collaboration's risk of bias tool, not all the articles adopted a good randomization method. None of the articles mentioned whether allocation concealment was performed, whether the blind method was used for subjects, whether the blind method was used for outcome evaluators, or whether selective reporting was used. The outcome indicators were complete, and none of them contained other risks of bias. Figures 2,3 show the risk of bias evaluation of the included studies.

\section{Stone clearance rate}

Seven articles $(8-13,15-17)$ mentioned the problem of the stone clearance rate of PCNL combined with negative pressure suction in the treatment of renal calculi. The metaanalysis showed that there was no significant difference in the stone clearance rate between the test group and the control group (OR $=1.21,95 \% \mathrm{CI}$ : $0.95,1.53 ; \mathrm{P}=0.118)$. As $\mathrm{I}^{2}=0.0 \%$, the fixed-effects model was used (see Figure 4). 
Table 1 Basic characteristics of the articles

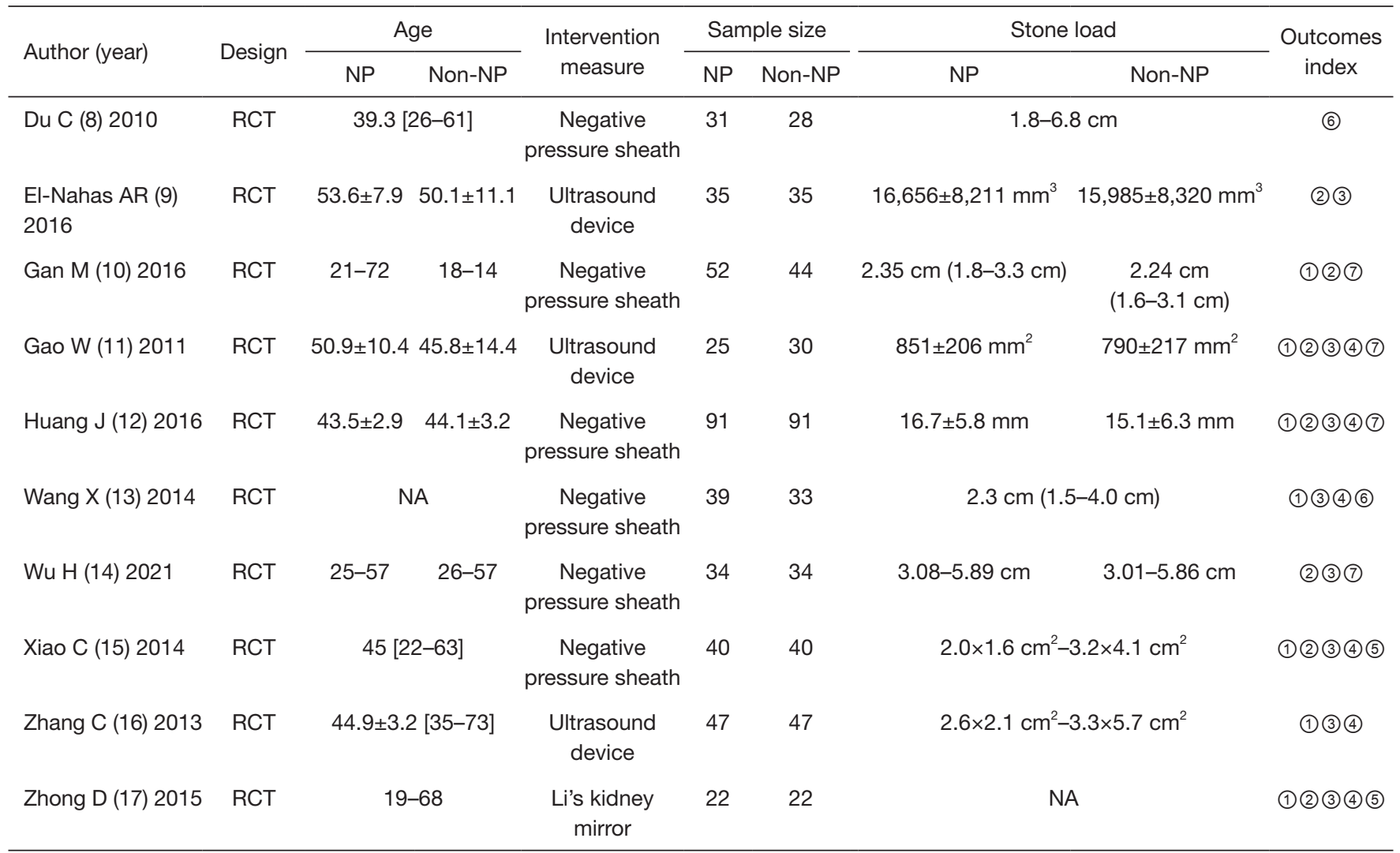

NP, negative pressure group; Non-NP, no negative pressure group; (1) Stone clearance rate; (2) Operation time; (3) Postoperative complications; (4) Postoperative fever; (5) Septic shock; (6) Endogenous pressure; (7) Amount of bleeding.

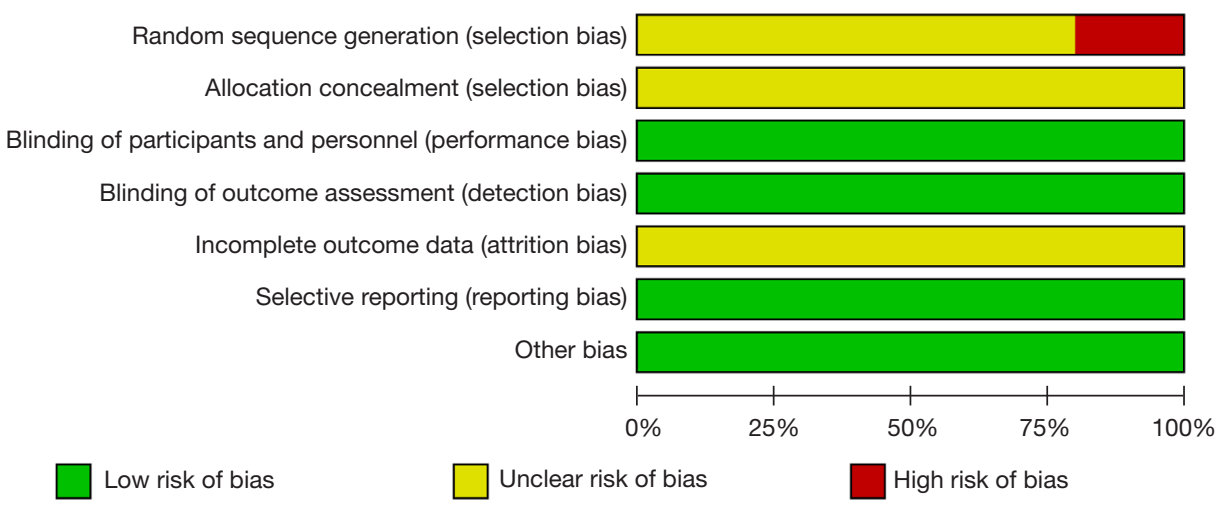

Figure 2 Risk of bias graph.

\section{Operation time}

Seven articles $(9-12,14,15,17)$ mentioned the operation time of PCNL combined with negative pressure suction in the treatment of renal calculi. The meta-analysis showed that the operation time of the test group was significantly shorter than that of the control group (SMD $=1.06,95 \%$ CI: 0.61 , $1.51 ; \mathrm{P}<0.01)$. The results showed significant heterogeneity $\left(\mathrm{I}^{2}=83.9 \%\right)$. After the sensitivity analysis, the articles were removed one by one, but this did not significantly reduce 


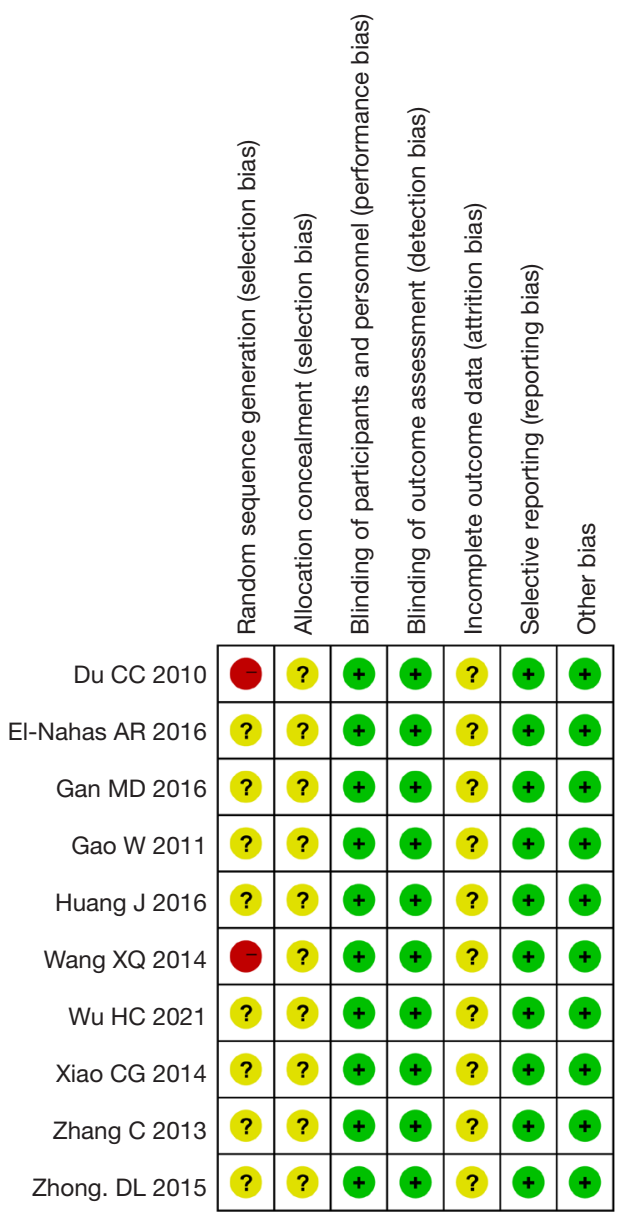

Figure 3 Risk or bias summary.

the heterogeneity. Thus, the random-effects model was selected (see Figures 5,6).

\section{Postoperative complications}

Eight articles $(9,11-17)$ mentioned problems related to the postoperative complications of PCNL combined with negative pressure suction in the treatment of renal calculi. The meta-analysis showed that the incidence rate of postoperative complications of the test group was significantly lower than that of the control group (OR $=0.45,95 \%$ CI: $0.30,0.68 ; \mathrm{P}<0.01)$. As $\mathrm{I}^{2}=16.2 \%$, the fixedeffects model was selected (see Figure 7).

\section{Postoperative fever}

Five articles $(11-13,15,17)$ mentioned the problem of postoperative fever of PCNL combined with negative pressure suction in the treatment of renal calculi. The metaanalysis showed that the postoperative fever of the test group was significantly better than that of the control group ( $\mathrm{OR}=0.40,95 \%$ CI: $0.23,0.71 ; \mathrm{P}=0.02)$. As $\mathrm{I}^{2}=0.0 \%$, the fixed-effects model was selected (see Figure 8).

\section{Septic shock}

Three articles (15-17) mentioned the problem of septic shock of PCNL combined with negative pressure suction in the treatment of renal calculi. The meta-analysis showed that there was no significant difference in the occurrence of septic shock between the test group and the control group ( $\mathrm{OR}=0.43$, 95\% CI: $0.11,1.70 ; \mathrm{P}=0.228)$. As $\mathrm{I}^{2}=0.0 \%$, the fixed-effects model was selected (see Figure 9).

\section{Blood loss volume}

Four articles $(10-12,14)$ mentioned the problem of blood loss in PCNL combined with negative pressure suction in the treatment of renal calculi. The meta-analysis showed that the intraoperative blood loss of the test group was significantly less than that of the control group (SMD $=2.42,95 \%$ CI: $1.32,3.52 ; \mathrm{P}<0.001)$. As $\mathrm{I}^{2}=93.8 \%$, the random-effects model was selected (see Figure 10).

\section{Test for heterogeneity}

Publication bias was tested using Egger's linear regression, and the results showed that there was no significant publication bias in any of outcome indicators except that of septic shock (see Table 2).

\section{Discussion}

The 2 indicators that best explain the difference between PCNL combined with negative pressure aspiration in the treatment of renal calculi and no negative pressure aspiration are operation time and blood loss. In the traditional treatment, it often takes a great deal of time to absorb the stone fragments, blood clots, and many floccules, which prolongs the operation time. However, a large number of reports $(9-12,14,15,17)$ have pointed out that when PCNL is combined with negative pressure suction, the operation time can be greatly shortened and the efficiency improved. This article did not obtain a positive result in relation to the stone clearance rate. It is 


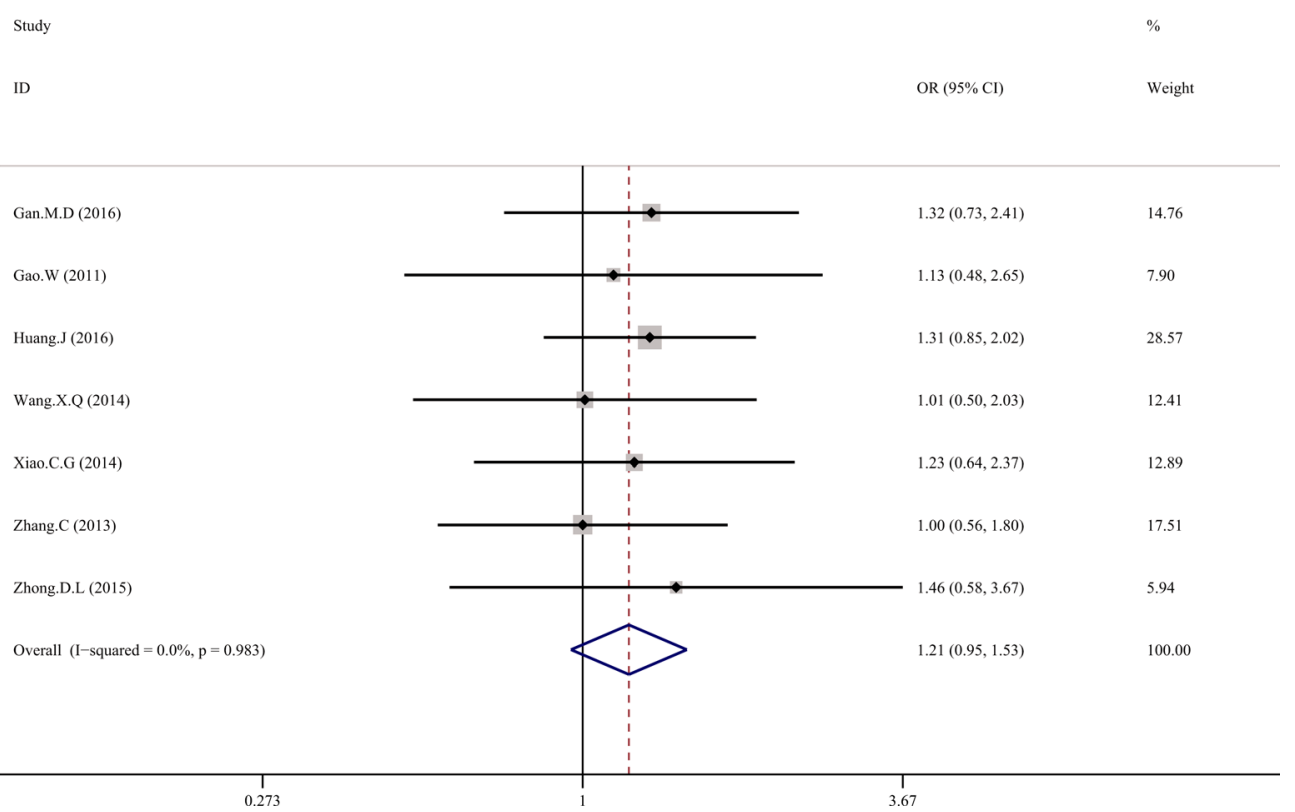

Figure 4 Pooled forest plot of the stone clearance rate results of PCNL combined with negative pressure suction in the treatment of renal calculi.

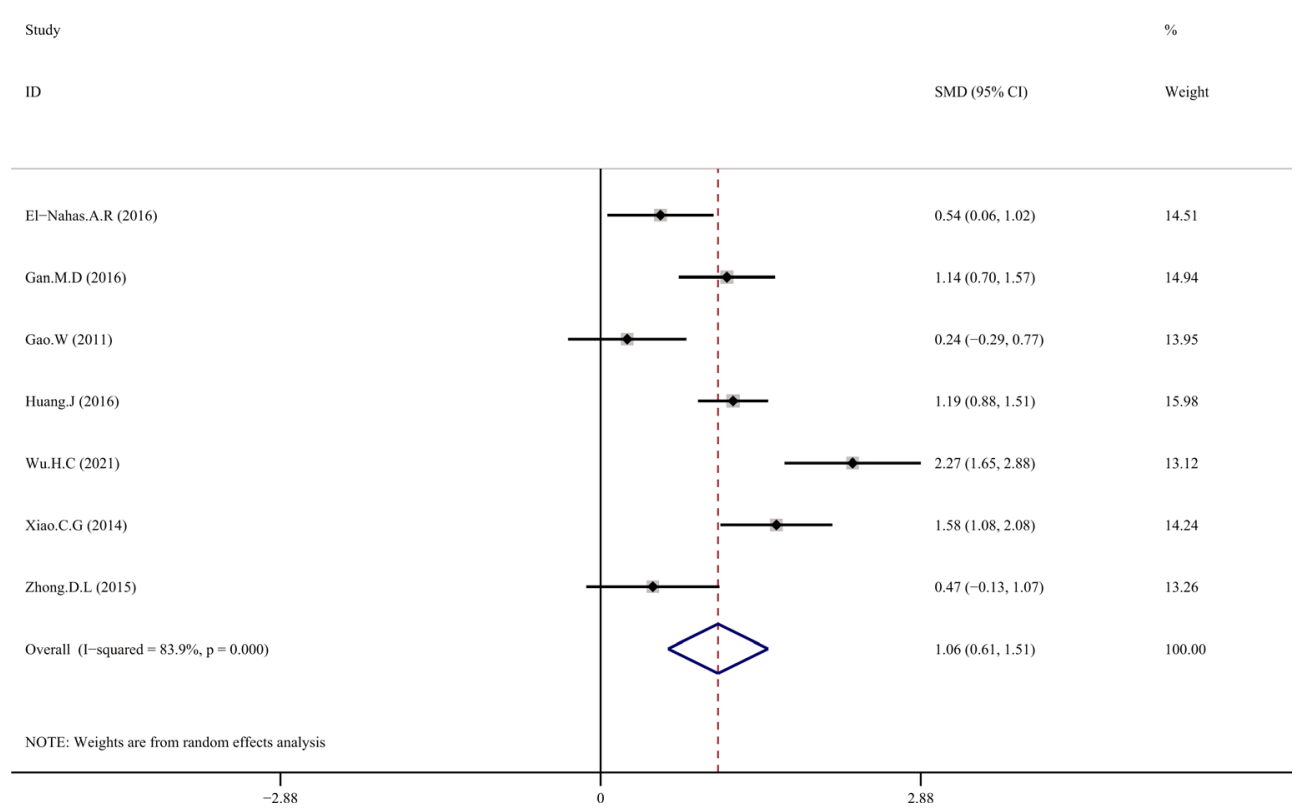

Figure 5 Pooled forest plot of the operation time results of PCNL combined with negative pressure suction in the treatment of renal calculi. PCNL, percutaneous nephrolithotomy.

speculated that while an operation with a large incision is not as fast as PCNL combined with negative pressure suction, it is already a mature technique, and the removal ability of stones using this technique has reached a relatively high level; thus, there is no great difference between the two. Yu et al. (4) pointed out that negative pressure suction can remove stone fragments, floccules, and blood clots more quickly than irrigation, basket, or forceps. It has 


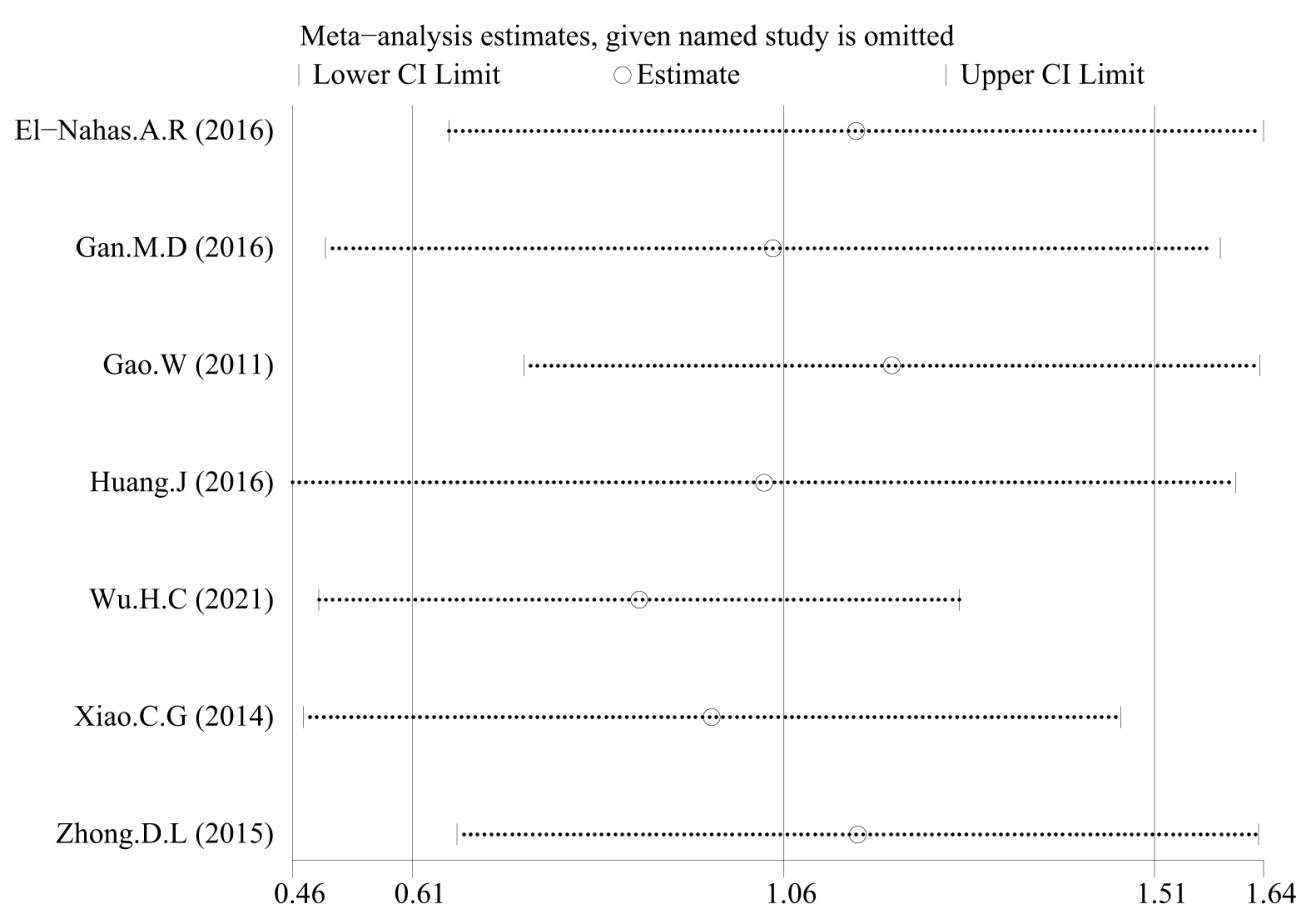

Figure 6 Sensitivity analysis.

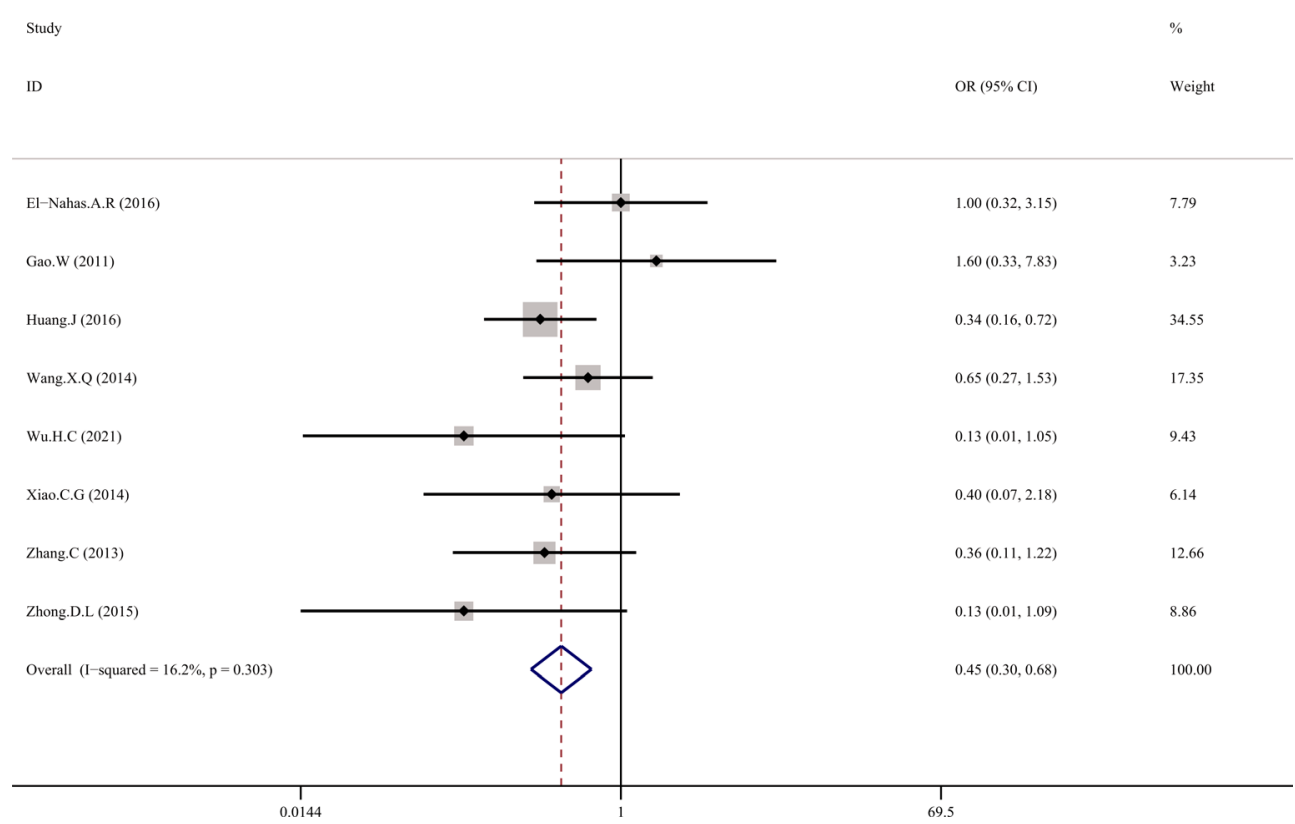

Figure 7 Forest plot of the postoperative complication results of PCNL combined with negative pressure suction in the treatment of renal calculi. PCNL, percutaneous nephrolithotomy.

been reported in the literature (9) that without a negative pressure suction device, stones need to be flushed out more finely, which prolongs the operation time. Some scholars have pointed out that the negative pressure device can reduce the displacement of stone fragments while absorbing stones, and can also suck out the pus in the calyces at the 


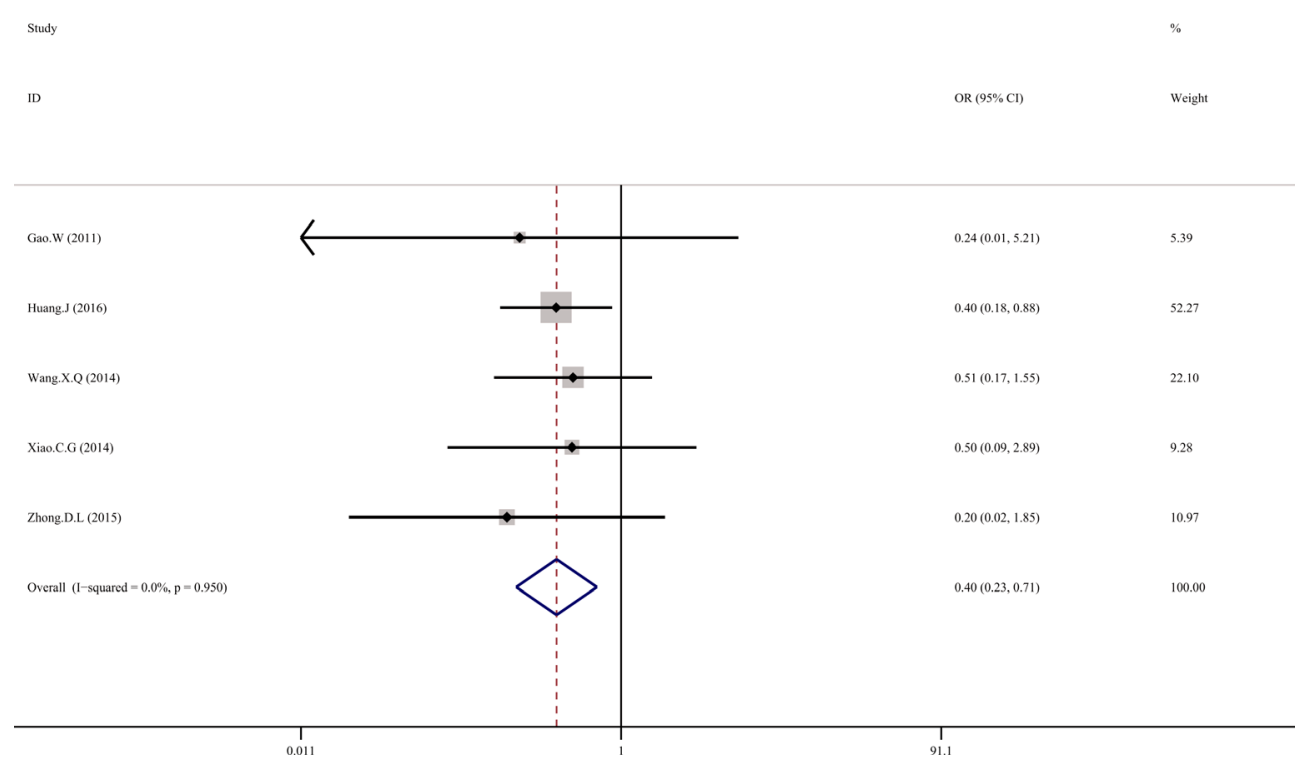

Figure 8 Forest plot of the postoperative fever results of PCNL combined with negative pressure suction in the treatment of renal calculi. PCNL, percutaneous nephrolithotomy.

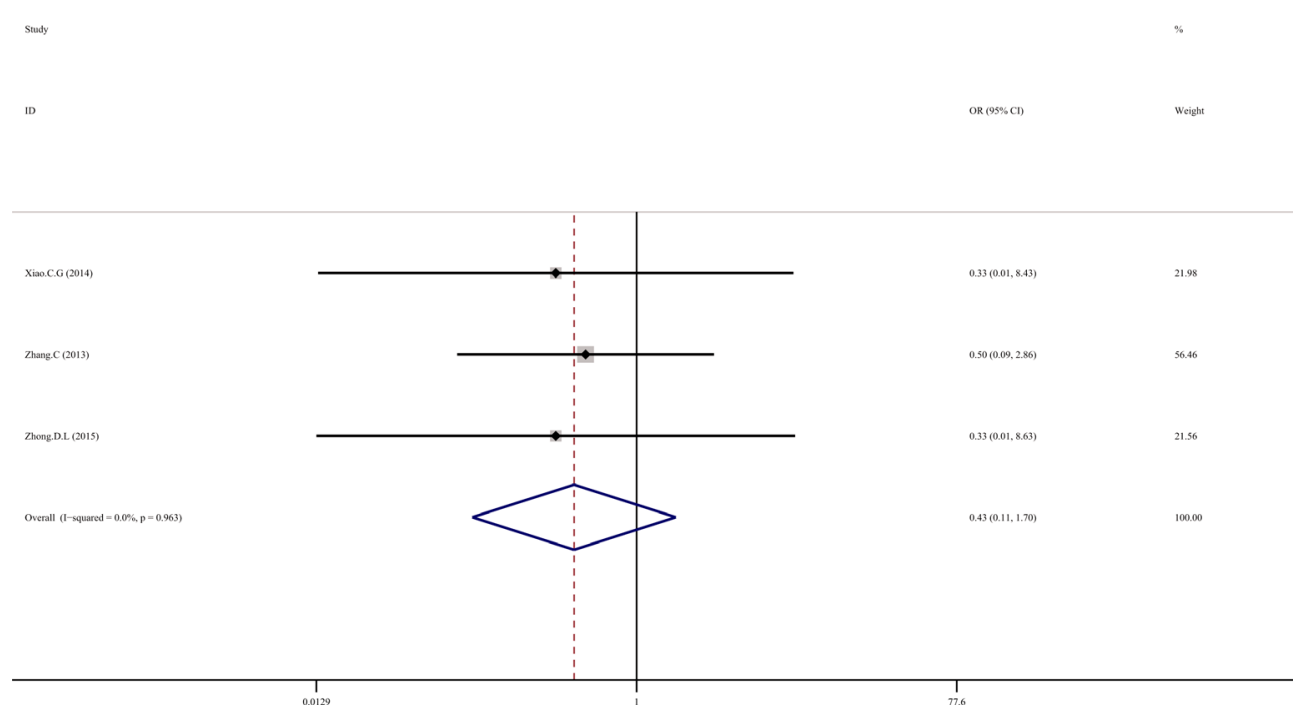

Figure 9 Pooled forest plot of the septic shock results of PCNL combined with negative pressure suction in the treatment of renal calculi. PCNL, percutaneous nephrolithotomy.

same time (18), reducing the time required for repeated washes.

Blood loss is an indicator used to evaluate a surgical technique objectively and visually shows the blood lost during surgery. In the case of the same incision, an increase in operation time will lead to an increase in blood loss (19). The same operation time, a small incision, and a small trauma not only contribute to the reduction of blood loss in patients, but are also more conducive to the postoperative recovery of patients. Thus, regardless of controlling for any variable, the progress of technology is evident when the amount of bleeding is reduced.

The occurrence of postoperative complications is also an important indicator for evaluating a technique. Some scholars (20) point out that when the intrapelvic pressure is too high, it will damage the integrity of the 


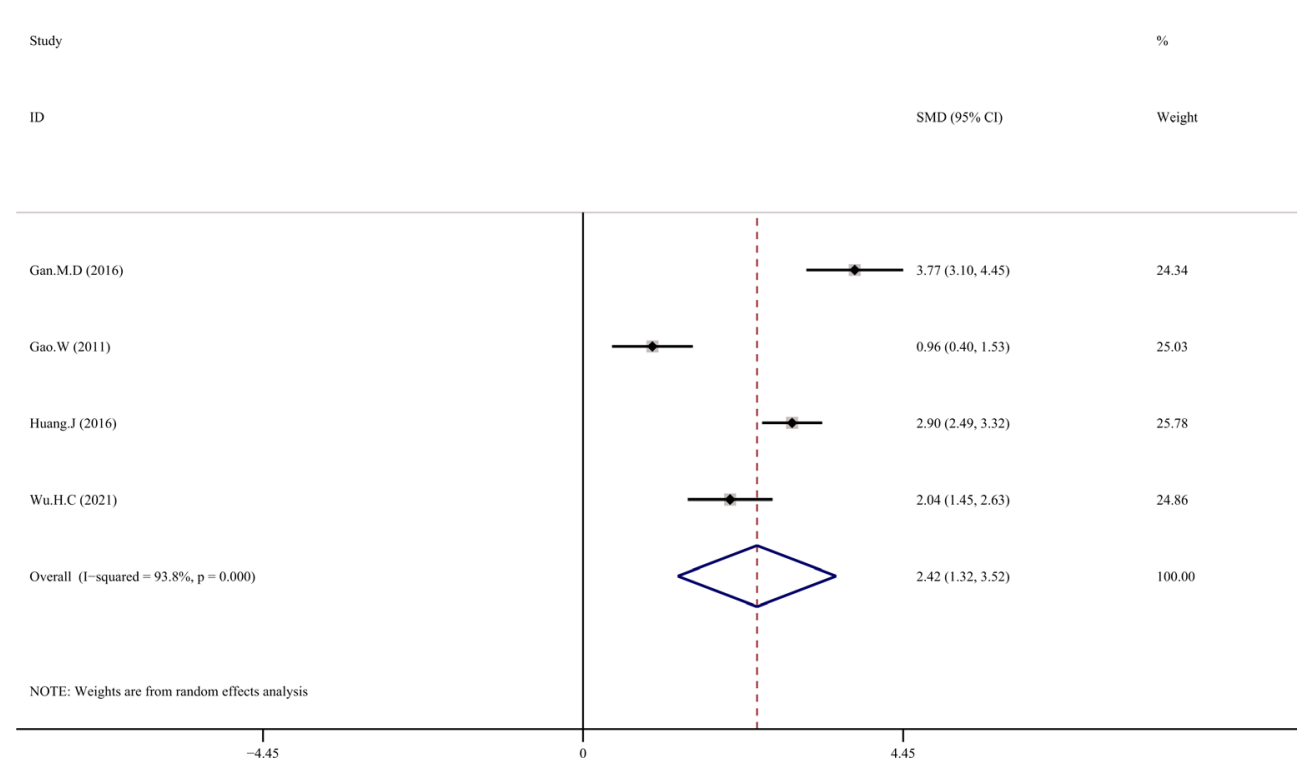

Figure 10 Pooled forest plot of the bleeding volume results of PCNL combined with negative pressure suction in the treatment of renal calculi. PCNL, percutaneous nephrolithotomy.

Table 2 Publication bias of outcome indicators

\begin{tabular}{lcc}
\hline Outcomes indicators & Amount & Egger's test P \\
\hline Stone clearance rate & 7 & 0.751 \\
Operation time & 7 & 0.910 \\
Postoperative & 8 & 0.829 \\
complications & 5 & 0.491 \\
Postoperative fever & 5 & $0.006^{\star}$ \\
Septic shock & 3 & 0.926 \\
Amount of bleeding & 4 & \\
\hline
\end{tabular}

*, $P<0.05$.

epithelial layer of the renal pelvis wall, exposing the veins and lymphatic system, resulting in renal parenchyma. However, the combination of negative pressure suction during surgery can significantly reduce intrapelvic pressure (8). After continuous practice and exploration, domestic and foreign guidelines have recommended percutaneous nephrolithotomy as the first-line treatment of kidney stones greater than $2 \mathrm{~cm}(21,22)$. Its increasing stone clearance rate, the decreasing trauma to patients, and the decreasing incidence rate of complications, have become an irreplaceable role in the urological urinary tract stone surgery method today. Due to the insufficient number of articles included in this study, this could not be proven at present. It is hoped that more RCTs will be conducted to provide relevant theoretical support for this conjecture in the future.

\section{Conclusions}

In summary, PCNL combined with negative pressure suction in the treatment of renal calculi significantly shortens the operation time and reduces surgical complications. There was high heterogeneity in some outcome indicators in this study. It may be related to different operator techniques and the different lithotripsy equipment used. It is hoped that more high-quality, large-sample size, multicenter RCTs will be conducted in the future to confirm the correctness of the relevant conclusions.

\section{Acknowledgments}

Funding: None.

\section{Footnote}

Reporting Checklist: The authors have completed the PRISMA reporting checklist. Available at https://tau. amegroups.com/article/view/10.21037/tau-21-1067/rc

Conflicts of Interest: All authors have completed the ICMJE 
uniform disclosure form (available at https://tau.amegroups. com/article/view/10.21037/tau-21-1067/coif). The authors have no conflicts of interest to declare.

Ethical Statement: The authors are accountable for all aspects of the work in ensuring that questions related to the accuracy or integrity of any part of the work are appropriately investigated and resolved.

Open Access Statement: This is an Open Access article distributed in accordance with the Creative Commons Attribution-NonCommercial-NoDerivs 4.0 International License (CC BY-NC-ND 4.0), which permits the noncommercial replication and distribution of the article with the strict proviso that no changes or edits are made and the original work is properly cited (including links to both the formal publication through the relevant DOI and the license). See: https://creativecommons.org/licenses/by-nc-nd/4.0/.

\section{References}

1. Lv J, Wang X, Zhao Z, et al. Meta-analysis of ureteral soft microscopy combined with negative pressure traction for the treatment of infectious kidney stones. The Chinese Journal of Endoscopy 2021;8:52-9.

2. Liu Y, Zhang Y. Meta-analysis of the treatment effects of three different minimally invasive kidney stones. Systems Medicine 2020;4:174-176+194.

3. Fernström I, Johansson B. Percutaneous pyelolithotomy. A new extraction technique. Scand J Urol Nephrol 1976;10:257-9.

4. Yu Y, Liao S, Wu Y, et al. Experimental study of renal injury by constant renal pressure pelvis perfusion. The Journal of Clinical Urology 2006;21:387-9.

5. Li X. Minimally invasive percutaneous nephrolithotomy. The Chinese Journal of Urology 2008;29:656.

6. Higgins JP, Altman DG, Gøtzsche PC, et al. The Cochrane Collaboration's tool for assessing risk of bias in randomised trials. BMJ 2011;343:d5928.

7. Chen D, Liu X, Chang B. Systematic evaluation and metaanalysis of risk factors for multiple resistant bacterial infections in diabetic foot ulcers. Journal of Hainan Medical College: 1-11[2021-11-27]. DOI:10.13210/j.cnki. jhmu.20210518.002.

8. Du C, Song L, Qin W, et al. Comparison study of internal pressure changes by lithotomy without negative pressure device. Chinese Journal of Cavoscopic Urology (Electronic
Edition) 2010;1:32-5.

9. El-Nahas AR, Elshal AM, El-Tabey NA, et al. Percutaneous nephrolithotomy for staghorn stones: a randomised trial comparing high-power holmium laser versus ultrasonic lithotripsy. BJU Int 2016;118:307-12.

10. Gan M, Li B, Ou Q, et al. Clinical analysis of standard percutaneous nephroscopy combined with negative pressure suction stone sheath for the treatment of kidney stones. Youjiang Medical 2016;2:156-9.

11. Gao W, Li X, He Y, et al. Comparative study of minimally invasive endoscopic ultrasonic lithotripsy system with double catheter and pneumatic ballistic lithotripsy for the treatment of cast renal stones. Chinese Journal of Cavoscopic Urology (Electronic Edition) 2011;6:448-452.

12. Huang J, Song L, Xie D, et al. A Randomized Study of Minimally Invasive Percutaneous Nephrolithotomy (MPCNL) with the aid of a patented suctioning sheath in the treatment of renal calculus complicated by pyonephrosis by one surgery. BMC Urol 2016;16:71.

13. Wang X, Zhang X, Xu Y, et al. Application of negative pressure traction in minimally invasive percutaneous nephroscopy holmium laser lithotripsy. Chinese Journal of Cavinal Surgery (Electronic Edition) 2014;6:482-4.

14. Wu $\mathrm{H}$, Zhang $\mathrm{M}$, Wang Q, et al. Clinical study of complicated renal stone extraction combined with negative pressure attraction. Modern Medicine and Health 2021;9:1530-2.

15. Xiao C, Li Z, Xiao L, et al. Application of self-made simple negative pressure attraction device in microplastic fistula percutaneous nephrectomy. Jiangxi Medicine 2014;12:1448-50.

16. Zhang C, Zhou Y, Lang J, et al. Transcutaneous percutaneous nephrolithotomy and ultrasonic lithotripsy for the treatment of complicated renal stones. Health Must Reading (X) 2013;12:192.

17. Zhong D, Wu Y, Mo G, et al. Effect of negative pressure attraction on fever after secondary percutaneous lithotomy in patients with pyogenes. The Journal of Clinical Urology 2015;4:339-341.

18. Song L, Chen Z, Liu T, et al. The application of a patented system to minimally invasive percutaneous nephrolithotomy. J Endourol 2011;25:1281-6.

19. Pu C, Wang J, Tang Y, et al. The efficacy and safety of percutaneous nephrolithotomy under general versus regional anesthesia: a systematic review and meta-analysis. Urolithiasis 2015;43:455-66.

20. Zhong $\mathrm{W}$, Zeng G. Intracelenoid pressure was measured 
during invasive minimally percutaneous nephrolithotomy.

Chinese Journal of Experimental Surgery 2011;28:121-3.

21.That Yanqun. Guide Manual for the Diagnosis and

Treatment of Urological Diseases in China: 2014 Edition.
The People's Health Press, 2014.

22.Türk C, Petř́k A, Sarica K, et al. EAU Guidelines on Interventional Treatment for Urolithiasis. Eur Urol 2016;69:475-82.

Cite this article as: Chen J, Cai X, Wang G, Chen X, Lin D. Efficacy and safety of percutaneous nephrolithotomy combined with negative pressure suction in the treatment of renal calculi: a systematic review and meta-analysis. Transl Androl Urol 2022;11(1):79-90. doi: 10.21037/tau-21-1067 